\title{
Out of the Chorus Line: What Makes 1E 1207.4-5209 a Unique Object?
}

Andrea De Luca, Patrizia Caraveo, Sandro Mereghetti, Michele Moroni

Istituto di Astrofisica Spaziale e Fisica Cosmica, Sezione di Milano "G.Occhialini" - CNR, Via Bassini 15, I-20133 Milano, Italy

Giovanni Bignami

Centre d'Etude Spatiale des Rayonnements, Toulouse, France Università degli Studi di Pavia, Via Ugo Bassi, 6 - Pavia, Italy

Roberto Mignani

European Southern Observatory, D-85740, Garching, Germany

\begin{abstract}
The discovery of deep spectral features in the X-ray spectrum of 1E 1207.4-5209 has pushed this Isolated Neutron Star (INS) out of the chorus line, since no other INS has shown significant features in its X-ray continuum. On 2002 Aug, XMM-Newton devoted a two-orbit target-of-opportunity observation to this target, with the aim of better understanding the nature of such spectral features using much improved statistics. Indeed, the $260 \mathrm{ksec}$ observation yielded 360000 photons from $1 \mathrm{E} 1207.4-5209$, allowing for a very sensitive study of the temporal and spectral behavior of this object.
\end{abstract}

\section{Introduction}

Neutron star atmosphere models predicted the presence of absorption features depending on atmospheric composition, but high quality spectra, collected both by Chandra and by XMM-Newton, have not yielded evidence for any feature (see Pavlov, Zavlin \& Sanwal 2002 and Becker \& Aschenbach 2002 for recent reviews). INS spectra are well fitted by one or more black-body curves with, possibly, a power law contribution at higher energies, but with no absorption or emission features. The spectrum of $1 \mathrm{E} 1207.4-5209$, on the contrary, is dominated by two broad absorption features seen at 0.7 and $1.4 \mathrm{keV}$, both by Chandra (Sanwal et al. 2002) and XMM-Newton (Mereghetti et al. 2002). To better understand the nature of such features, $X M M$-Newton devoted two orbits, for a total observing time of $257303 \mathrm{~s}$, to $1 \mathrm{E} 1207.4-5209$. In the two MOS EPIC cameras the source yielded 74600 and 76700 photons in the energy range $0.2-3.5 \mathrm{keV}$, while the pn camera recorded 208000 photons, time-tagged to allow for timing studies. Analysis of this long observation, while confirming the two phase-dependent absorption lines at 0.7 and $1.4 \mathrm{keV}$, unveiled a statistically significant third line at $\sim 2.1 \mathrm{keV}$, as well as a possible fourth feature at $2.8 \mathrm{keV}$. The nearly 1:2:3:4 
ratio of the line centroids, as well as the phase variation, naturally following the pulsar magnetic field rotation, strongly suggest that such lines are due to cyclotron resonance scattering (Bignami et al. 2003). A recent software release, based on a better characterization of the EPIC instrument, prompted us to revisit the data. While the spectral analysis results confirm and strengthen the conclusions of Bignami et al. (2003), the temporal and spatial analysis yielded interesting new results which we shall briefly outline (see De Luca et al. 2004 for details).

\section{Timing Analysis}

After converting the arrival times of the 208000 pn photons to the solar system barycenter, we searched the period range from 424.12 to $424.14 \mathrm{~ms}$ using both a folding algorithm with eight phase bins and the Rayleigh test. The best period value and its uncertainty $(P=424.13076 \pm 0.00002 \mathrm{~ms})$ were determined following the procedure outlined in Mereghetti et al. (2002). Comparing the new period measurement of $1 \mathrm{E}$ 1207.4-5209 with that obtained with Chandra in 2000 Jan (Pavlov et al. 2002), we obtain a period derivative $\dot{P}=(1.4 \pm 0.3) \times$ $10^{-14}$. However, Figure 1 (left) shows that the $\dot{P}$ value rests totally on the first Chandra period measurement. Using only the three most recent values, the period derivative is unconstrained. Thus, we cannot exclude that the observed spin-down, based on only a few sparse measurements, may be affected by glitches or Doppler shifts induced by orbital motion. Questioning the object's $P$ would have far-reaching consequences for the understanding of 1E 1207.4-5209 since the serious discrepancy between the pulsar characteristic age $\left(\tau_{c} \sim 5 \times 10^{5} \mathrm{yr}\right)$ and the SNR age ( $\left.\tau_{\mathrm{SNR}} \sim 7 \mathrm{kyr}\right)$ is entirely based on the value inferred from the measurements summarized in Figure 1 (left).

To study the energy dependence of the pulse profile, we divided the data into four channels with approximately 52000 counts each: $0.2-0.52 \mathrm{keV}, 0.52$ $0.82 \mathrm{keV}, 0.82-1.14 \mathrm{keV}$ and $1.14-3.5 \mathrm{keV}$. The pulse profiles in the different energy ranges (Fig. 1, right) show a broad, nearly sinusoidal shape, with a pulsed fraction varying from $\sim 3 \%$ to $\sim 11 \%$ in the four energy intervals. It is worth noting that the minimum pulsed fraction is found in the $0.2-0.52 \mathrm{keV}$ energy range, the only portion of the spectrum free from absorption lines. Indeed, Figure 1 (right) is an independent confirmation of the findings of Bignami et al. (2003) who ascribe the source pulsation to the absorption lines' phase variation.

Finally, comparing the shapes of the light curves of Figure 1 (right), we see for the first time a phase shift of nearly $90^{\circ}$ between the profile in the lowest energy range $(<0.52 \mathrm{keV})$ and those at higher energies.

\section{Optimizing the X-ray Position}

To derive the sky coordinates of $1 \mathrm{E}$ 1207.4-5209 we computed independently for the MOS1 and MOS2 cameras the boresight correction to be applied to the default EPIC astrometry. We used the Guide Star Catalog II (GSC-II ${ }^{1}$ ) to select,

\footnotetext{
${ }^{1}$ http://www-gsss.stsci.edu/gsc/gsc2/GSC2home.htm.
} 

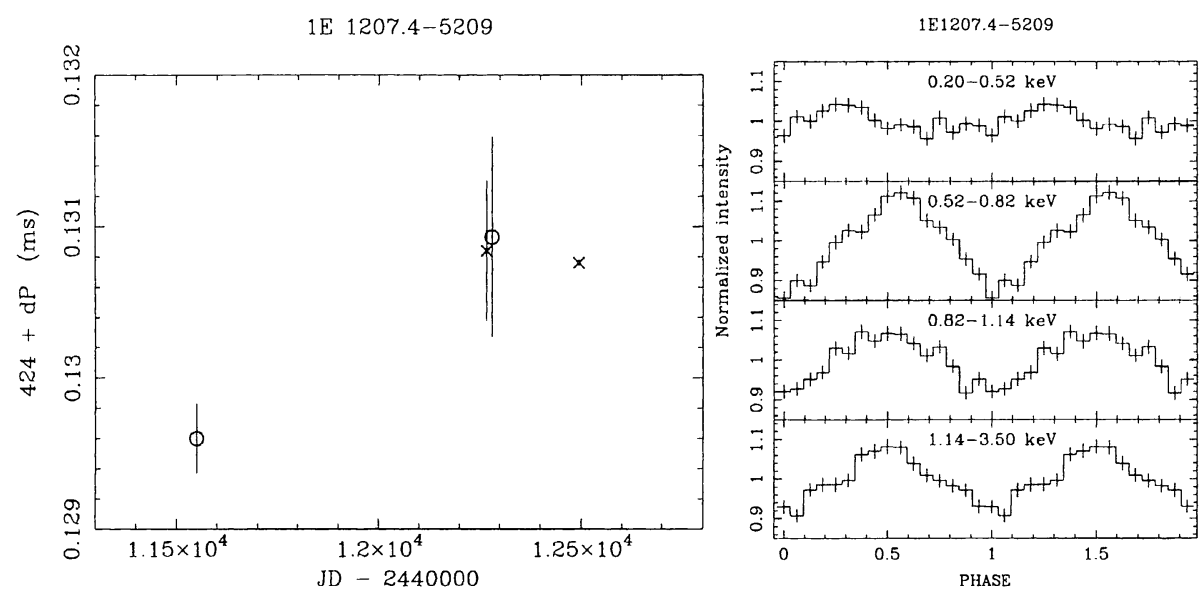

Figure 1. Left panel: Period history of 1E 1207.4-5209. Measurements are represented by circles (Chandra) and crosses (XMMNewton). Right panel: Folded light curve of 1E 1207.4-5209 in four energy ranges. Fitting the pulse profiles with a function consisting of a constant plus a sinusoid, we obtain pulsed fraction values of $(2.7 \pm 0.7) \%$ in the $0.2-0.52 \mathrm{keV}$ energy range, $(11.1 \pm 0.7) \%$ for $0.52-0.82 \mathrm{keV}$, $(7.1 \pm 0.7) \%$ for $0.82-1.14 \mathrm{keV}$, and $(7.1 \pm 0.7) \%$ for $1.14-3.5 \mathrm{keV}$.

among our $\sim 200$ serendipitous detections, 6 sources with a stellar counterpart to be used to correct the EPIC astrometry. The rms error between the refined Xray and GSC-II positions is $\sim 1$ arcsec in each coordinate. The resulting MOS1 position of $1 \mathrm{E} 1207.4-5209$ is $\mathrm{RA}=12^{\mathrm{h}} 10^{\mathrm{m}} 00^{\mathrm{s}} .91$, Decl. $=-52^{\circ} 26^{\prime} 28^{\prime \prime} .8$ (J2000) with an overall error radius of $1^{\prime \prime} 5$. The MOS2 position is $\mathrm{RA}=12^{\mathrm{h}} 10^{\mathrm{m}} 00^{\mathrm{s}} .84$, Decl. $=-52^{\circ} 26^{\prime} 27^{\prime \prime} 6(\mathrm{~J} 2000)$, with an uncertainty of $1^{\prime \prime} .5$, fully consistent with the MOS1 coordinates.

\section{Search for the Optical Counterpart}

The field of 1E 1207.4-5209 was observed with the 8.2-meter UT-1 Telescope (Antu) of the ESO VLT (Paranal Observatory). Observations were performed with the the FOcal Reducer and Spectrograph 1 (FORS1) instrument. Images were acquired through the Bessel $V$ and $R$ filters for a total integration time of $\sim 2$ and $3 \mathrm{hr}$, respectively. Figure 2 shows the inner portion of the combined FORS1 $V$-band image centered on the target position, with the MOS1 and MOS2 error circles superimposed. A faint object (marked with the two ticks in Fig. 2) is detected just outside the southern edge of the MOS1 error circle and showed variability during the time span covered by our observations. In any case, its position falls more than $2^{\prime \prime}$ away from the intersection of the MOS1/MOS2 error circles, which we regard as the most probable region.

No candidate counterpart is detected within the MOS1/MOS2 error circles down to $R \sim 27.1$ and $V \sim 27.3$, which we assume as upper limits on the optical flux of $1 \mathrm{E}$ 1207.4-5209. For the X-ray-derived interstellar absorption 


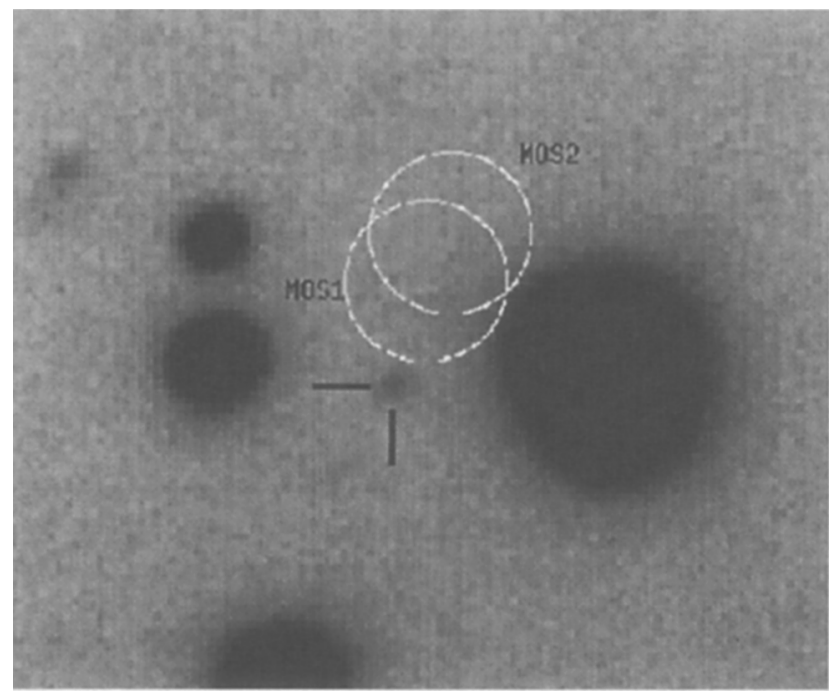

Figure 2. FORS1 $V$-band image centered on the target position, with the MOS1 and MOS2 error circles ( 2 " radius) superimposed.

$\left(A_{V}=0.65\right)$ and a distance of $2 \mathrm{kpc}$, the measured upper limits rule out any hypothetical "normal" stellar companion other than a very low-mass main sequence star. If we assume that $1 \mathrm{E} 1207.4-5209$ is indeed isolated, we can derive a neutron star optical luminosity $\leq 3.4 \times 10^{28} \mathrm{ergs} \mathrm{s}^{-1}$ or $\leq 4.6 \times 10^{-6}$ of its rotational energy loss, a value similar to those of middle-aged INSs. Since the VLT flux upper limits are $\geq 100$ higher than the extrapolation of the XMM-Newton blackbody (De Luca et al. 2004), they cannot constrain the optical spectrum.

\section{References}

Bignami, G. F., Caraveo, P. A., De Luca, A., \& Mereghetti, S. 2003, Nature, 423,725

Becker, W., \& Aschenbach, B. 2002, in MPE Rep. 278, Neutron Stars, Pulsars, and Supernova Remnants, eds. W. Becker, H. Lesch, \& J. Trümper, (Garching: MPE), p. 64

De Luca, A., Mereghetti, S., Caraveo, P. A., Moroni, M., Mignani, R. P., \& Bignami, G. F. 2004, A\&A, 418, 625

Mereghetti, S., De Luca, A., Caraveo, P. A., Becker, W., Mignani, R., \& Bignami, G. F. 2002, ApJ, 581, 1280

Pavlov, G. G., Zavlin, V. E., \& Sanwal, D. 2002, in MPE Rep. 278, Neutron Stars, Pulsars, and Supernova Remnants, eds. W. Becker, H. Lesch, \& J. Trümper, (Garching: MPE), p. 273

Pavlov G. G., Zavlin, V. E., Sanwal, D., \& Trümper, J. 2002, ApJ, 569, L95

Sanwal D., Pavlov G. G., Zavlin V. E., \& Teter, M. A. 2002, ApJ, 574, L61 